\title{
Supernovae at the Extremes
}

\author{
Ke-Jung Chen ${ }^{1,2, *}$ \\ ${ }^{1}$ Division of Theoretical Astronomy, National Astronomical Observatory of Japan, Tokyo, \\ Japan \\ ${ }^{2}$ Department of Astronomy \& Astrophysics, University of California, Santa Cruz, California, \\ USA \\ *EACOA Fellow, email: ken.chen@nao.ac.jp
}

\begin{abstract}
During a supernova explosion, fluid instabilities are generated because the star is in a hydrodynamically unstable situation, which is like the effects of stirring a fire or blowing air into a hot grill. The resulting mixing of the supernova ejecta may be observable. Here, we briefly discuss the multidimensional simulations of supernovae from very massive stars.
\end{abstract}

Keywords. supernovae; nuclear reactions; hydrodynamics; instabilities

\section{Summary}

When massive stars reach the end of their lives, some die as supernovae (SNe), the most powerful explosions in the Universe. SNe are a frontier topic in modern astrophysics with immense scientific richness for theoretical and observational explorations. Very massive stars from 90-140 $M_{\odot}$ can encounter the pair production instability (PI) after central carbon burning instead of collapsing to black holes. The PI is able to trigger multiple massive outbursts from the star. Collisions between the ejected shells can power very luminous optical transits known as pulsational pair-instability supernovae (PPI SNe). This phenomenon may explain superluminous SNe, such as SN2006gy and SN2007bi, which defy traditional interpretation as core-collapse SNe because of their extraordinary brightness. Multidimensional simulations of PPI SNe from Chen et al. (2014a) revealed that a significant amount of mixing occurs during the collisions of the shells. Because the collisions are extremely luminous, mixing may easily alter the observational signatures of PPI SNe.

In stars with masses of 140-260 $M_{\odot}$, the PI occurs just after central helium burning, causing rapid contraction of the core and triggering explosive oxygen and silicon burning that completely disrupts the star, leaving no compact remnant. These events are known as pair-instability supernovae (PI SNe). Chen et al. (2014b) performed the first 2D simulations of initial collapse, bounce and explosion to study mixing in Pop III PI SNe. Because their models followed the explosion from the onset of collapse, they could capture dynamical instabilities in the flow seeded by core contraction and explosive burning, not just the subsequent expansion of the SNe shock through the star as done in previous simulations. Their results found that the degree of mixing prior to shock breakout from the surface of the star is sensitive to its pre-supernova structure. Besides, explosive burning does somewhat exacerbate the growth of fluid instabilities but the resulting mixing does not extend into the ${ }^{56} \mathrm{Ni}$ layer or dredge it up to lower depths. Previous 1D light curve calculations of Pop III PI SNe that assume the ${ }^{56} \mathrm{Ni}$ is mixed throughout the ejecta therefore may predict premature ${ }^{56} \mathrm{Ni}$ rebrightening.

Previous stellar evolution models showed that primordial stars over $300 M_{\odot}$ simply collapse to black holes. But Chen et al. (2014c) discovered that some supermassive Pop III stars do not die quietly. Those in a narrow window in mass around $55,000 M_{\odot}$ can 
explode in the most energetic thermonuclear explosions in the Universe. Unlike normal $\mathrm{SNe}$ or PI SNe, the explosions of supermassive stars are triggered by general relativistic instabilities that begin during central helium burning, which leads to a runaway collapse of the core that ignites explosive helium burning. The energy released can be up to $10^{55}$ erg, or 10,000 times the energy of Type Ia SNe. These cataclysmic events completely unbind the star and indeed destroy the protogalaxies that gave birth to them.

\section{Acknowledgements}

K.C. acknowledges the support of EACOA Fellowship from the East Asian Core Observatories Association. Work at UCSC has been supported by an IAU-Gruber Fellowship, the DOE HEP Program (DE-SC0010676) and the NASA Theory Program (NNX14AH34G). Numerical simulations are supported by the Minnesota Supercomputing Institute (MSI), the National Energy Research Scientific Computing Center (NERSC), and the Center for Computational Astrophysics (CfCA) at National Astronomical Observatory of Japan (NAOJ).

\section{References}

Chen, K.-J., Woosley, S., Heger, A., Almgren, A., \& Whalen, D. J. 2014a, ApJ, 792, 28

Chen, K.-J., Heger, A., Woosley, S., Almgren, A., \& Whalen, D. J. 2014b, ApJ, 792, 44

Chen, K.-J., Heger, A., Woosley, S., et al. 2014c, ApJ, 790, 162 